\title{
Neutrophil Elastase Inhibitor (Sivelestat), may be a Promising Therapeutic Option for Management of Acute Lung Injury/Acute Respiratory Distress Syndrome or Disseminated Intravascular Coagulation in COVID-19
}

\author{
Adeleh Sahebnasagh ${ }^{1}$, Fatemeh Saghafi ${ }^{2}$, Mohammadreza Safdari ${ }^{1}$, Masoud Khataminia ${ }^{3}$, \\ Afsaneh Sadremomtaz ${ }^{4}$, Hassan Rezai Ghaleno ${ }^{5}$, Mahdi Bagheri ${ }^{6}$, Mohammad Sadegh \\ Bagheri ${ }^{6}$, Solomon Habtemariam ${ }^{7}$, and Razieh Avan ${ }^{8}$ \\ ${ }^{1}$ North Khorasan University of Medical Sciences \\ ${ }^{2}$ Shahid Sadoughi University of Medical Sciences and Health Services \\ ${ }^{3}$ Tehran University of Medical Sciences \\ ${ }^{4}$ University of Groningen \\ ${ }^{5}$ Lorestan University of Medical Sciences \\ ${ }^{6}$ Baqiyatallah University of Medical Sciences \\ ${ }^{7}$ University of Greenwich \\ ${ }^{8}$ Birjand University of Medical Sciences
}

May 18, 2020

\begin{abstract}
This article summarizes the effects of sivelestat on ALI/ARDS or ARDS with coagulopathy, both of which are frequently seen in patients with COVID-19. The emergence of the novel 2019-nCoV infection has been a global pandemic. The following databases were searched to identify relevant literature concerning empirical evidence: The Cochrane library, Pubmed, Medline, EMBASE from 1980 through March 2020. COVID-19 patients are more susceptible to thromboembolic diseases including DIC. In this connection, various studies have emphasized on the role of neutrophil elastase (NE) in the development of DIC in patients with ARDS and sepsis. It has been shown that NE inhibition by sivelestat mitigates ALI through amelioration of alveolar epithelium and vascular endothelium injuries as well as reversing the activated neutrophil-mediated increased vascular permeability. Sivelestat is a selective NE inhibitor has not been evaluated for its possible therapeutic effects against SARS-CoV2 infection and/or COVID-19. Nevertheless, based on its promising beneficial effects in underlying complications of COVID-19, sivelestat could be considered as a promising treatment for the management of ALI/ARDS or coagulopathy in patients with COVID-19.
\end{abstract}

\section{KEYWORDS}

COVID-19, ALI/ARDS, Coagulopathy, Sivelestat, Neutrophil elastase inhibitor

\section{INTRODUCTION}

The little known respiratory infection by a novel coronavirus 2019-nCoV (or SARS-CoV-2) in December 2019 in Wuhan, China has now spread to become a global pandemic. On 11 February 2020, the World Health Organization (WHO) named the disease as COVID-19, while the International Committee on Taxonomy of Viruses renamed the virus based on phylogeny, and taxonomy, as severe acute respiratory syndrome coronavirus- 2 
(SARS-CoV-2) (1). At the time of preparing this manuscript, WHO reported over two million confirmed positive cases of COVID-19 and the number of global death now exceeds 157, 847 (Coronavirus disease 2019 (COVID-19) Situation Report - 91. https://www.who.int/docs/default-source/coronaviruse/situationreports/20200420-sitrep-91-covid-19.pdf?sfvrsn=fcf0670b_4). The death rate of COVID-19 is estimated to be $2 \%(2)$, which is less than SARS and the Middle East Respiratory Syndrome (MERS), but SARS-CoV-2 is proven to be more contagious (3). The resulting hospitalization, death and measure of social distancing leading to complete shutdown of the human socioeconomic activity brought up unprecedented level of global concern.

SARS-CoVs, MERS-CoVs and SARS-CoV-2 are members of the beta-coronavirus family (4). Recent reports have shown that SARS-CoV-2 has 79\% nucleotide similarity to SARS-CoV and 51.8\% to MERS-CoV (5). In addition to non-structural proteins, the viral genome encodes structural proteins such as the membrane (M), envelope (E), nucleocapsid (N) and spike (S) proteins. Playing key role for coronavirus pathogenicity (6), these structural proteins are involved in viral entry into host cells and replication (7). The similarity of SARS$\mathrm{CoV}-2$ structural proteins with SARS-CoV was noted, implying similar entry mechanism through attachment to the host receptor, angiotensin-converting enzyme 2 (ACE2). For membrane fusion, the interaction between spike protein and ACE2 appears to be a critical step in a cascade of viral-host cell communications (7). The RNA-dependent RNA polymerase (RdRp) has a role for replication of SARS-CoV-2 RNA genome. The packing of new virions have been shown to be dependent on two polyproteins which are processed by $3 \mathrm{C}$-like and papain-like proteases (6).

Although the symptoms of COVID-19 could vary depending on age and underlying conditions (8). Most patients exhibit symptoms such as fever, dry cough, myalgia, tiredness, and diarrhea. People over the age of 60 and with past medical history of hypertension, diabetes, chronic obstructive pulmonary disease (COPD), cardiovascular, cerebrovascular, hepatic, renal, and gastrointestinal diseases are at higher risk for developing SARS-CoV-2 infection with a substantial mortality rate (6). Patients with COVID-19 are also more susceptible to thromboembolic diseases because of immobility, inflammation, hypoxia, and disseminated intravascular coagulation (DIC). In one study, the incidence of thrombotic complications in patients admitted to the intensive care unit (ICU) with COVID-19 was reported to reach $31 \%$ (9). In this connection, hyperfibrinolysis related to an increase in D-dimer levels was observed in $97 \%$ of patients with COVID-19 at the time of hospital admission and it continued to increase in all patients before death. Fibrin degradation products also significantly increased throughout the course of the disease. In severe cases of COVID-19 or in dying patients, a significant drop in platelet levels was observed (6). Numerous reports also outlined that COVID-19 patients may experience a severe form of the disease during a few days, which is often manifested as an acute lung injury/acute respiratory distress syndrome (ALI/ARDS), respiratory failure, heart failure, or sepsis (8). In animal model of SARS-CoV and MERS-CoV, the significant levels of inflammatory and immune responses cause "cytokine storm", and apoptosis of epithelial and endothelial cells. This is followed by an increase in vascular permeability and leakage, abnormal $\mathrm{T}$ cell and macrophages responses, and ALI/ARDS that could lead to death (10).

In patients with COVID-19, the inflammatory cytokine storm is closely associated with the development and progression of ARDS (11). In these patients, the high levels of expression of inflammatory cytokines such as interleukin (IL)-1 $\beta$, interferon (IFN)- $\gamma$-induced protein (IP-10), and monocyte chemoattractant protein 1 (MCP-1) could result in activated T-helper-1 (Th1) response. Furthermore, SARS-CoV-2 infection, unlike that of SARS-CoV, induces the release of T-helper-2 (Th2) cytokines, such as IL-4 and IL-10, with inflammation suppression ability. Further comprehensive studies are however essential to determine the Th1 and Th2 cells responses in COVID-19. When compared to hospitalized COVID-19 patients at general wards, those in the ICU appears to show higher level of granulocyte colony-stimulating factor, IP-10, MCP-1, macrophage inflammatory protein-1A, and tumor necrosis factor-alpha (TNF- $\alpha$ ). Several studies emphasized this positive correlation between cytokine storm and COVID-19 severity (12). Furthermore, an elevated level of IL-6 was shown to be a predictor of poor outcome in severe COVID-19 with pneumonia and ARDS (13). Hence, the inflammatory cytokine storm play key role both for the development of ARDS and extra-pulmonary organ failure (11). 
ARDS is a severe type of acute lung injury characterized by massive infiltration of neutrophils, monocytes and lymphocytes. The diffuse bilateral edema followed by reduced lung compliance, alveolar damage, and bronchoalveolar lumen hyaline deposition in ARDS result in hypoxic respiratory failure. Degranulating neutrophils have a key role in the development of capillary injury, leakage, and hyaline deposition. These events may progress ARDS to a more fatally diffuse alveolar damage (14). The key role of neutrophils in the pathogenesis of ALI/ARDS has also been shown in animal and clinical studies. Histological assay on autopsy samples of ARDS patients have illustrated a significant accumulation of polymorphonuclear cells (PMN) including neutrophils in the damaged alveoli and in the interstitial tissues (15). Till now, there is no specific treatment for ARDS except for supportive care using low-tidal volume ventilators that limit trans-pulmonary pressures (16). Unfortunately, low-tidal volume ventilation might result in hypercapnia and higher hospital mortality (17).

Nuclear Factor kappa-light-chain-enhancer of activated B cells (NF- $\chi \mathrm{B})$ is a basic transcription factor that is essential for the expression of inflammation-related genes such as inducible nitric oxide synthase (iNOS) and inflammatory cytokines (18). In patients with ARDS, the activation of NF- $x \mathrm{~B}$ leads to increased expression of immunoregulatory and pro-inflammatory cytokines (19).

Viral infection has also the potential to induce the production of oxidized products or oxidative stress that aggravates the inflammation-mediated COVID-19 pathology. For example, oxidized low density lipoprotein under SARS-induced ALI activate the innate immune response leading to overproduction of IL-6 in alveolar macrophages via the Toll-like receptor 4 (TLR4)/NF- $x$ B signaling pathway (20). With active viral infection, the retinoic acid-inducible gene I (RIG-I) also senses viral RNA and triggers signaling cascades, adaptor proteins (MAVS and TRAF), and different transcription factors (NF- $x \mathrm{~B}$ and IRF3/IRF7) at host pattern recognition receptors (PRRs). This constitutes the beginning of transcription of antiviral type I interferon and pro-inflammatory cytokines (21).

As major components of inflammatory responses to endothelial injury, neutrophils have proteolytic, and pro-apoptotic properties through the action of several enzymes (22). Among them is the serine protease, neutrophil elastase (NE), which has antimicrobial properties due to its ability to degrade phagocytosed pathogens (23). It also contributes to inflammation by increasing vascular permeability (24) and induction of the release of pro-inflammatory cytokines such as IL-6 and IL-8 (25). In this regard, NE is needed for the function of neutrophil both under regulated inflammatory response and tissue damage during sepsis. Under normal physiological conditions, the function of $\mathrm{NE}$ is rigorously regulated by endogenous protease inhibitors (25). Under exaggerated inflammatory conditions, however, NE is enabled to attack the endothelial barrier and infiltrate to bronchoalveolar space, since protease inhibitors are inactivated by neutrophil oxidants (26). Thus, excessive activity of NE may lead to tissue damage and remodeling in a number of pulmonary diseases such as community acquired pneumonia, ventilator-associated pneumonia, exacerbated COPD, cystic fibrosis, bronchiectasis, and ALI/ARDS (27). Furthermore, in those patients with ALI/ARDS, plasma levels of NE are significantly higher in comparison to healthy subjects (28). The significant level of proteolytic activity of NE was also observed in bronchoalveolar lavage (BAL) of ARDS patients (29).

Sivelestat, also known in the scientific literature as ONO-5046, is a selective, reversible and competitive neutrophil elastase inhibitor. Hence, it has no effect on the function of other proteases in the body (30). Its protective effects in attenuating ALI/ARDS has been described in several models of lung injury. In different preclinical and animal models of lung injury, sivelestat mitigated the elevation in the lung vasculature permeability, pulmonary artery pressure (PAP), increase in the lung tissue wet or dry weight ratio, and neutrophil count (31-33). Furthermore, sivelestat improved pathogen clearance, the decrease in $\mathrm{PaO} 2$, and prevented digestion of surfactant protein $\mathrm{D}(34,35)$.

\section{Methods}

The following databases were searched to identify relevant literature concerning empirical evidence: The Cochrane library, Pubmed, Medline, EMBASE from 1980 through March 2020.

\section{Results}


It has been shown that NE inhibition by sivelestat mitigate ALI through amelioration of alveolar epithelium and vascular endothelium injuries as well as reversing the activated neutrophil-mediated increased vascular permeability (31). In a clinical study on patients with ARDS and systemic inflammatory response syndrome, continuous infusion of sivelestat has been shown to significantly improve pulmonary function, as indicated by increased in $\mathrm{PaO} 2 / \mathrm{FIO} 2$ ratio, shortened duration of mechanical ventilation time and length of ICU care. However, the mortality rate did not differ in comparison to control group (36). In another clinical (phase III double blinded) trial with 230 ALI patients, sivelestat was shown to increase recovery rates of the pulmonary functional parameters, reduced the duration of mechanical ventilator and better weaning rates, and shortened ICU length of stay by almost half. However, this selective NE inhibitor was not able to reduce 30 -day survival rate. In this study, only around $20 \%$ of death occurred due to respiratory failure. This may explain why survival rate between the two arms of the study did not reach a significant value (37).

There are several studies indicating that sivelestat increases ventilator-free days and survival in patients with ALI/ARDS by inhibition of the overstretch-induced signaling pathway and neutrophil chemotaxis (38-40). During mechanical ventilation (MV), the risk of ALI is increased due to over-activation of neutrophil elastase and myeloperoxidase. Furthermore, phosphorylation of c-Jun NH2-terminal kinase (JNK) is increased in alveolar type 2 epithelial cells (41). There is a direct relationship between inhibition of JNK and prevention of over-ventilation lung injury. JNK is also critical in induction of apoptosis during stress responses (42). The protective effects of sivelestat against MV was evaluated in animal model of mice where sivelestat (100 mg/kg, intraperitoneally) or saline was administered 30 minute before ventilation. Under 4 hours of MV with high tidal volume of $20 \mathrm{~mL} / \mathrm{kg}$, sivelestat prevented histopathological MV induced-lung damage, decreased lung tissue wet to dry weight ratio, and suppressed the serum and bronchoalveolar lavage fluid levels of macrophage-inflammatory protein 2 (MIP-2), IL-6 and TNF- $\alpha$. By inhibition of neutrophil chemotaxis, sivelestat also normalized the phosphorylation level of JNK and attenuated apoptotic changes in pneumocytes after the MV-induced ALI (43).

In a retrospective study of sivelestat in 110 patients with ALI and sepsis, sivelestat significantly increased the number of ventilator free days and $\mathrm{PaO} 2 / \mathrm{FIO} 2$, especially in those patients with baseline procalcitonin levels of [?] $0.5 \mathrm{ng} / \mathrm{mL}$ (40). In another multicenter, prospective study using 164 mechanically ventilated ARDS patients with high wet-to-dry lung weight ratio, the efficacy of sivelestat was compared to the control group. In this study, sivelestat increased ventilator-free days with no significant effect on 28-day mortality (39).

In a phase IV open-label, non-randomized, multi-center clinical trial for the treatment of ALI associated with SIRS on 581 patients, sivelestat was continuously administered intravenously at a dose of $0.2 \mathrm{mg} / \mathrm{kg} / \mathrm{h}$ for 2 weeks. The results of this study showed a significant higher ventilator-free day, ICU discharge rate and early weaning from mechanical ventilator with sivelestat compared to control group. Furthermore, those patients received sivelestat had a substantially higher 180-day survival than control patients. The results of this clinical trial emphasized on the clinical efficacy of this NE inhibitor in this group of patients (44).

During ARDS and cytokine storm, the serum levels of TNF- $\alpha$, IL-6, high mobility group box 1 (HMGB1) protein, and NO synthesis from iNOS, which contribute to the host's inflammatory responses, rise significantly (45-47). As indicated in the preceding texts, the NF- $x \mathrm{~B}$ signaling pathway regulates the expression of these inflammatory factors $(48,49)$. Furthermore, NE-mediated chemotaxis to the lung potently induces epithelial cells to produce inflammatory cytokines and macrophages to MCP-1 (50). It has been demonstrated that sivelestat decreases NF- $\chi \mathrm{B}$ pathway stimulation, and inhibits the secretion of HMGB1 from macrophages through inhibition of I $\chi \mathrm{B}$ kinase phosphorylation (51). Moreover, the serum levels of TNF- $\alpha$, IL-6, HMGB1, and NO were shown to significantly decrease following administration of sivelestat. This selective NE inhibitor could also substantially reduce the level of MCP-1 mRNA in macrophages during ischemia-reperfusion injury (52).

As outlined above, COVID-19 patients are more susceptible to thromboembolic diseases including DIC. In this connection, various studies have emphasized on the role of NE in the development of DIC in patients with ARDS and sepsis. In a study on 167 septic patients with ARDS and DIC, sivelestat was administered 
upon admission to ICU and continued for 5 days. The results showed that sivelestat improved lung injury score, $\mathrm{PaO} 2 / \mathrm{FIO} 2$ ratio, DIC score, and ICU length of stay and survival rate when compared to the control group (53).

In another study on 142 ARDS patients with DIC, the efficacy of sivelestat alone, recombinant human soluble thrombomodulin (rhTM) alone, combination therapy of sivelestat and rhTM or untreated control were evaluated and compared to each other. The results were very promising, indicating that combination therapy with sivelestat and rhTM significantly increased the 60-day patient survival, mechanical ventilatorfree days, and better PIO2/FIO2 ratio and DIC score (54).

\section{CONCLUSION}

In conclusion, this article has given an overview of the effects of sivelestat on ALI/ARDS or ARDS with coagulopathy, both of which are frequently seen in patients with COVID-19. This selective NE inhibitor has not been evaluated for its possible therapeutic effects against SARS-CoV-2 infection and/or COVID-19. Nevertheless, based on its promising beneficial effects in underlying complications of COVID-19, sivelestat could be considered as a promising treatment for the management of ALI/ARDS or coagulopathy in patients with COVID-19. In the end, we think that testing the possible efficacy of this therapeutic modality in this group of patients could help in developing new strategies for combating COVID-19.

\section{ACKNOWLEDGMENTS}

We appreciate all practitioner, health care provider and researchers who is giving a fight against the 2019$\mathrm{nCoV}$ pandemic.

\section{CONFLICT OF INTEREST}

The authors declare that there are no conflicts of interest.

\section{REFERENCES:}

1. Lai C-C, Shih T-P, Ko W-C, Tang H-J, Hsueh P-R. Severe acute respiratory syndrome coronavirus 2 (SARS-CoV-2) and corona virus disease-2019 (COVID-19): the epidemic and the challenges. Int J Antimicrob Agents. 2020:105924.

2. Xu Z, Shi L, Wang Y, Zhang J, Huang L, Zhang C, et al. Pathological findings of COVID-19 associated with acute respiratory distress syndrome. Lancet Respir Med. 2020;8(4):420-2.

3. Wang D, Hu B, Hu C, Zhu F, Liu X, Zhang J, et al. Clinical characteristics of 138 hospitalized patients with 2019 novel coronavirus-infected pneumonia in Wuhan, China. JAMA. 2020;323(11):1061-9.

4. Chen J. Pathogenicity and transmissibility of 2019-nCoV - a quick overview and comparison with other emerging viruses. Microbes Infect. 2020.

5. Ren L-L, Wang Y-M, Wu Z-Q, Xiang Z-C, Guo L, Xu T, et al. Identification of a novel coronavirus causing severe pneumonia in human: a descriptive study. Chin Med J. 2020.

6. Ji H-L, Zhao R, Matalon S, Matthay MA. Elevated plasmin (ogen) as a common risk factor for COVID-19 susceptibility. Physiol Rev. 2020.

7. Shanmugaraj B, Siriwattananon K, Wangkanont K, Phoolcharoen W. Perspectives on monoclonal antibody therapy as potential therapeutic intervention for Coronavirus disease-19 (COVID-19). Asian Pac J Allergy Immunol. 2020;38(1):10-8.

8. Zhang R, Wang X, Ni L, Di X, Ma B, Niu S, et al. COVID-19: Melatonin as a potential adjuvant treatment. Life Sci. 2020:117583.

9. Klok F, Kruip M, van der Meer N, Arbous M, Gommers D, Kant K, et al. Incidence of thrombotic complications in critically ill ICU patients with COVID-19. Thromb Res. 2020. 
10. Channappanavar R, Perlman S, editors. Pathogenic human coronavirus infections: causes and consequences of cytokine storm and immunopathology. Semin Immunopathol; 2017: Springer.

11. Ye Q, Wang B, Mao J. Cytokine Storm in COVID-19 and Treatment. J Infect. 2020.

12. Huang C, Wang Y, Li X, Ren L, Zhao J, Hu Y, et al. Clinical features of patients infected with 2019 novel coronavirus in Wuhan, China. Lancet. 2020;395(10223):497-506.

13. Cao X. COVID-19: immunopathology and its implications for therapy. Nat Rev Immunol. 2020:1-2.

14. Kast R. Dapsone as treatment adjunct in ARDS. Exp Lung Res. 2020.

15. Kambas K, Markiewski MM, Pneumatikos IA, Rafail SS, Theodorou V, Konstantonis D, et al. C5a and TNF- $\alpha$ up-regulate the expression of tissue factor in intra-alveolar neutrophils of patients with the acute respiratory distress syndrome. J Immunol. 2008;180(11):7368-75.

16. Moore HB, Barrett CD, Moore EE, McIntyre RC, Moore PK, Talmor DS, et al. Is There a Role for Tissue Plasminogen Activator (tPA) as a Novel Treatment for Refractory COVID-19 Associated Acute Respiratory Distress Syndrome (ARDS)? J Trauma Acute Care Surg. 2020.

17. Liu X, Liu X, Xu Y, Xu Z, Huang Y, Chen S, et al. Ventilatory Ratio in Hypercapnic Mechanically Ventilated Patients with COVID-19 Associated ARDS. Am J Respir Crit Care Med. 2020(ja).

18. Singleton KD, Beckey VE, Wischmeyer PE. Glutamine prevents activation of NF- $\varkappa \mathrm{B}$ and stress kinase pathways, attenuates inflammatory cytokine release, and prevents acute respiratory distress syndrome (ARDS) following sepsis. Shock. 2005;24(6):583-9.

19. Moine P, McIntyre R, Schwartz MD, Kaneko D, Shenkar R, Le YT, et al. NF-kappaB regulatory mechanisms in alveolar macrophages from patients with acute respiratory distress syndrome. Shock (Augusta, Ga). 2000;13(2):85-91.

20. Imai Y, Kuba K, Neely GG, Yaghubian-Malhami R, Perkmann T, van Loo G, et al. Identification of oxidative stress and Toll-like receptor 4 signaling as a key pathway of acute lung injury. Cell. 2008;133(2):23549.

21. Li Z, Li X, Huang Y-Y, Wu Y, Zhou L, Liu R, et al. FEP-based screening prompts drug repositioning against COVID-19. bioRxiv. 2020.

22. Aikawa N, Kawasaki Y. Clinical utility of the neutrophil elastase inhibitor sivelestat for the treatment of acute respiratory distress syndrome. Ther Clin Risk Manag. 2014;10:621.

23. Segal AW. How neutrophils kill microbes. Annu Rev Immunol. 2005;23:197-223.

24. Ma Y, Yang X, Chatterjee V, Meegan JE, Beard Jr RS, Yuan SY. Role of neutrophil extracellular traps and vesicles in regulating vascular endothelial permeability. Front Immunol. 2019;10:1037.

25. Kawabata K, Hagio T, Matsuoka S. The role of neutrophil elastase in acute lung injury. Eur J Pharmacol. 2002;451(1):1-10.

26. Sandhaus RA, Turino G. Neutrophil elastase-mediated lung disease. COPD. 2013;10(sup1):60-3.

27. Polverino E, Rosales-Mayor E, Dale GE, Dembowsky K, Torres A. The role of neutrophil elastase inhibitors in lung diseases. Chest. 2017;152(2):249-62.

28. Kodama T, Yukioka H, Kato T, Kato N, Hato F, Kitagawa S. Neutrophil elastase as a predicting factor for development of acute lung injury. Intern Med. 2007;46(11):699-704.

29. McGuire W, Spragg R, Cohen A. Cochrane: Studies on the pathogenesis of the adult respiratory distress syndrome. J Clin Invest. 1982;69:543-53. 
30. Pu S, Wang D, Liu D, Zhao Y, Qi D, He J, et al. Effect of sivelestat sodium in patients with acute lung injury or acute respiratory distress syndrome: a meta-analysis of randomized controlled trials. BMC Pulm Med. 2017;17(1):148.

31. Miyazaki Y, Inoue T, Kyi M, Sawada M, Miyake S, Yoshizawa Y. Effects of a Neutrophil Elastase Inhibitor (ONO-5046) on Acute Pulmonary Injury Induced by Tumor Necrosis Factor Alpha (TNF $\alpha$ ) and Activated Neutrophils in Isolated Perfused Rabbit Lungs. Am J Respir Crit Care Med. 1998;157(1):89-94.

32. Kubo K, Kobayashi T, Hayano T, Koizumi T, Honda T, Sekiguchi M, et al. Effects of ONO5046, a specific neutrophil elastase inhibitor, on endotoxin-induced lung injury in sheep. J Appl Physiol. 1994;77(3):1333-40.

33. Sakamaki F, Ishizaka A, Urano T, Sayama K, Nakamura H, Terashima T, et al. Effect of a specific neutrophil elastase inhibitor, ONO-5046, on endotoxin-induced acute lung injury. Am J Respir Crit Care Med. 1996;153(1):391-7.

34. Hagio T, Matsumoto S, Nakao S, Abiru T, Ohno H, Kawabata K. Elastase inhibition reduced death associated with acid aspiration-induced lung injury in hamsters. Eur J Pharmacol. 2004;488(1-3):173-80.

35. Hagio T, Kishikawa K, Kawabata K, Tasaka S, Hashimoto S, Hasegawa N, et al. Inhibition of neutrophil elastase reduces lung injury and bacterial count in hamsters. Pulm Pharmacol Ther. 2008;21(6):884-91.

36. Okayama N, Kakihana Y, Setoguchi D, Imabayashi T, Omae T, Matsunaga A, et al. Clinical effects of a neutrophil elastase inhibitor, sivelestat, in patients with acute respiratory distress syndrome. J Anesth. 2006;20(1):6-10.

37. Tamakuma S, Ogawa M, Aikawa N, Kubota T, Hirasawa H, Ishizaka A, et al. Relationship between neutrophil elastase and acute lung injury in humans. Pulm Pharmacol Ther. 2004;17(5):271-9.

38. Hashimoto S, Okayama Y, Shime N, Kimura A, Funakoshi Y, Kawabata K, et al. Neutrophil elastase activity in acute lung injury and respiratory distress syndrome. Respirology. 2008;13(4):581-4.

39. Tagami T, Tosa R, Omura M, Fukushima H, Kaneko T, Endo T, et al. Effect of a selective neutrophil elastase inhibitor on mortality and ventilator-free days in patients with increased extravascular lung water: a post hoc analysis of the PiCCO Pulmonary Edema Study. J Intensive Care. 2014;2(1):67.

40. Miyoshi S, Hamada H, Ito R, Katayama H, Irifune K, Suwaki T, et al. Usefulness of a selective neutrophil elastase inhibitor, sivelestat, in acute lung injury patients with sepsis. Drug Des Dev Ther. 2013;7:305.

41. Uhlig S. Ventilation-induced lung injury and mechanotransduction: stretching it too far? Am J Physiol Lung Cell Mol Physiol. 2002;282(5):L892-L6.

42. Janssen-Heininger YM, Persinger RL, Korn SH, Pantano C, McElhinney B, Reynaert NL, et al. Reactive nitrogen species and cell signaling: implications for death or survival of lung epithelium. Am J Respir Crit Care Med. 2002;166(supplement_1):S9-S16.

43. Sakashita A, Nishimura Y, Nishiuma T, Takenaka K, Kobayashi K, Kotani Y, et al. Neutrophil elastase inhibitor (sivelestat) attenuates subsequent ventilator-induced lung injury in mice. Eur J Pharmacol. 2007;571(1):62-71.

44. Aikawa N, Ishizaka A, Hirasawa H, Shimazaki S, Yamamoto Y, Sugimoto H, et al. Reevaluation of the efficacy and safety of the neutrophil elastase inhibitor, Sivelestat, for the treatment of acute lung injury associated with systemic inflammatory response syndrome; a phase IV study. Pulm Pharmacol Ther. 2011;24(5):549-54.

45. Bhatia M, Moochhala S. Role of inflammatory mediators in the pathophysiology of acute respiratory distress syndrome. J Pathol. 2004;202(2):145-56. 
46. Medzhitov R, Janeway CA. Decoding the patterns of self and nonself by the innate immune system. Science. 2002;296(5566):298-300.

47. van der Poll T, van Deventer SJ. Cytokines and anticytokines in the pathogenesis of sepsis. Infect Dis Clin North Am. 1999;13(2):413-26.

48. Liu SF, Malik AB. NF- $x$ B activation as a pathological mechanism of septic shock and inflammation. Am J Physiol Lung Cell Mol Physiol. 2006;290(4):L622-L45.

49. Blackwell TS, Christman JW. The role of nuclear factor- $\chi$ B in cytokine gene regulation. Am J Respir Cell Mol Biol. 1997;17(1):3-9.

50. Yamaguchi Y, Akizuki E, Ichiguchi O, Matsumura F, Goto M, Miyanari N, et al. Neutrophil elastase inhibitor reduces neutrophil chemoattractant production after ischemia-reperfusion in rat liver. Gastroenterology. 1997;112(2):551-60.

51. Adcock I. Transcription factors as activators of gene transcription: AP-1 and NF-kappa B. Monaldi Arch Chest Dis. 1997;52(2):178-86.

52. Hagiwara S, Iwasaka H, Hidaka S, Hasegawa A, Noguchi T. Neutrophil elastase inhibitor (sivelestat) reduces the levels of inflammatory mediators by inhibiting NF-kB. Inflamm Res. 2009;58(4):198-203.

53. Hayakawa M, Katabami K, Wada T, Sugano M, Hoshino H, Sawamura A, et al. Sivelestat (selective neutrophil elastase inhibitor) improves the mortality rate of sepsis associated with both acute respiratory distress syndrome and disseminated intravascular coagulation patients. Shock. 2010;33(1):14-8.

54. Miyoshi S, Ito R, Katayama H, Dote K, Aibiki M, Hamada H, et al. Combination therapy with sivelestat and recombinant human soluble thrombomodulin for ARDS and DIC patients. Drug Des Dev Ther. 2014;8:1211. 